\title{
Empyema and Cavitation After Dental Procedure
}

\author{
Isaac Akkad ${ }^{\mathrm{a}, \mathrm{b}}$, Alberto Ortiz ${ }^{\mathrm{a}}$, Precious Macauley ${ }^{\mathrm{a}}$, Paurush Ambesh ${ }^{\mathrm{a}}$
}

\begin{abstract}
Thoracic complications following oropharyngeal procedures have been reported in medical literature. Aspiration of foreign bodies and gastric contents, upper airway obstruction, bacterial endocarditis, descending necrotizing mediastinitis, Lemierre syndrome, and pneumomediastinum are among the most commonly reported. In this case report, we present a 67-year-old Caucasian male without significant comorbidities found to have a left lower lobe empyema and a right upper lobe cavitary lesion after having a tooth extraction 4 months prior. The organisms found within the lesions are normal constituents of the oral flora. In patients presenting with intrathoracic symptoms within the context of recent dental procedures, the clinician should be aware of procedure-related complications that although uncommon could be life-threatening.
\end{abstract}

Keywords: Cavitary pulmonary disease; Empyema; Dental procedures

\section{Introduction}

Multiple conditions can be associated with the development of a pleural empyema. Pulmonary infection, surgery, trauma, esophageal perforation, septicemia, and complications of chest tube placement are among the most common [1]. Little has been reported about the association of pleural empyema following dental procedures $[2,3]$. Non-tuberculous pleural empyema is most commonly associated with streptococcus, staphylococcus, and bacteroides genus, although a wide spectrum of microorganisms have been identified [4]. The combination of both a cavitary lesion by Actinomyces israelii and pleural empyema by Streptococcus gordonii after a dental procedure has not been previously reported. An increasing awareness of these types of infections may prompt the development of primary prevention strategies as well as help expedite the diagnosis, minimizing undesirable outcomes.

Manuscript accepted for publication October 26, 2016

${ }^{a}$ Department of Medicine, Maimonides Medical Center, Brooklyn, NY 11219, USA

${ }^{b}$ Corresponding Author: Isaac Akkad, Department of Medicine, Maimonides Medical Center, Brooklyn, NY 11219, USA.

Email: iakkad@maimonidesmed.org

doi: http://dx.doi.org/10.14740/jmc2683w

\section{Case Report}

A 67-year-old Caucasian male with a past medical history of essential hypertension and previous prostatic cancer, successfully treated with resection and radiotherapy, presented to the emergency department (ED) complaining of an insidious onset of dry cough, subjective fevers, pleuritic chest pain, and shortness of breath for the past couple of weeks. His medications included a thiazide diuretic and an angiotensin receptor blocker. The patient denied ever smoking, alcohol, or illicit drug use, occupational exposures, or any recent travel history. He had visited his primary care doctor twice prior to presentation to the ED and finished an antibiotic course with levofloxacin for suspected community-acquired pneumonia. On physical exam, the patient was found to have good oral hygiene and no evidence of current dental infections. On cardiovascular exam, no JVD, friction rub, or murmurs were appreciated. On respiratory exam, the patient had dullness to percussion on the left lower lobe with crackles, decreased breath sounds on auscultation over same focus, and unremarkable right lung exam. There were no chest wall deformities, cyanosis, or accessory muscle use evidenced on inspection. There were no other pertinent positive physical findings. In the ED, patient was afebrile with oxygen $\left(\mathrm{O}_{2}\right)$ saturation of $97 \%$ room air and other vital signs where normal. The initial laboratory results showed an increased white blood cell count (WBC) of 18.9 with $90 \%$ neutrophil predominance and reactive thrombocytosis. $\mathrm{ABG}$ revealed a $\mathrm{pH}$ of 7.42, $\mathrm{pCO}_{2}$ of $44, \mathrm{HCO}_{3}{ }^{-}$of 25 , lactic acid of 3.3, and $\mathrm{pO}_{2}$ of 85 . No other electrolyte or metabolic abnormalities were noted. Chest X-ray (Fig. 1) showed a left pleural effusion with compressive atelectasis.

CT scan of the chest with IV contrast showed a cavitary lesion in the right upper lobe with irregular borders measuring $12.3 \mathrm{~mm}$, initially thought to reflect septic emboli or cavitary metastases. A left posterior lower pleural space empyema was also found (Figs. 2 and 3).

Broad-spectrum antibiotics were initiated and chest tube placement took place. Pleural drainage was abundant, malodorous, and viscous. The appropriate pleural studies confirmed the exudative nature of the effusion and cultures grew grampositive Streptococcus gordonii. Patient remained afebrile and hemodynamically stable throughout hospitalization with improvement in symptoms.

The cavitary lesion was a challenge. Initially we ruled out tuberculosis, endocarditis, and active oropharyngeal infection foci. There was a concern for malignancy. A small biopsy was taken for analysis, which showed tissue necrosis 


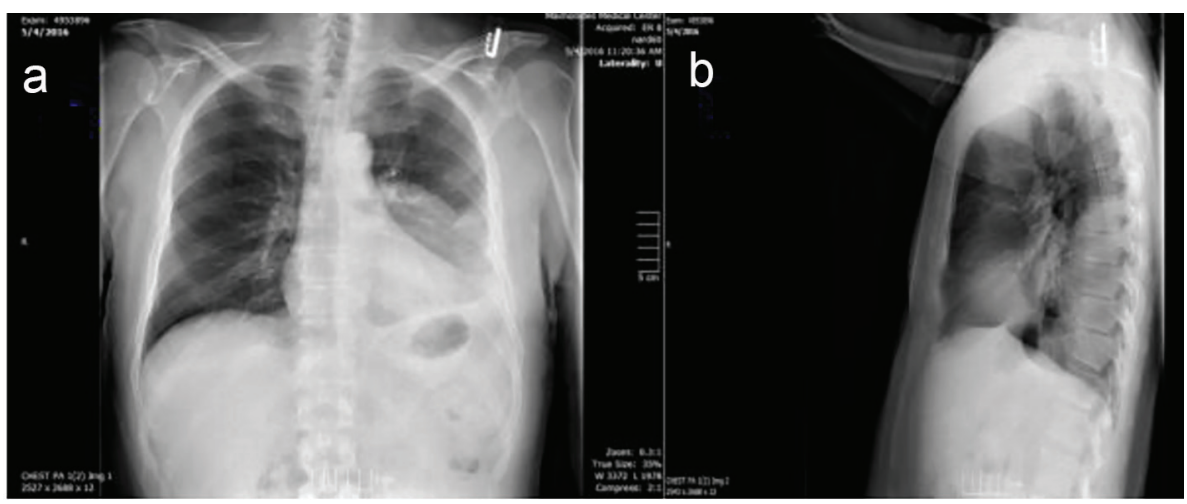

Figure 1. (A) Posterior-anterior and (B) lateral views on initial chest X-ray with findings of left pleural effusion and left lower lobe infiltrate.

with yellowish sulfur granules and filamentous gram-positive fungal-like pathogens confirming Actinomyces spp., susceptible to beta-lactams. Appropriate antibiotic course took place and patient was asymptomatic 6 weeks after hospital discharge.

\section{Discussion}

Pleural effusions can develop as a consequence of increased hydrostatic pressure or decreased oncotic pressure $[5,6]$. Host factors predisposing patients to empyema include pneumonia and parapneumonic effusions, as well as contiguous infections of the esophagus, mediastinum, and subdiaphragmatic areas [7, 8]. Pleural effusions are nutritionally rich culture media in which WBC defenses are severely impaired [9]. In overtly healthy adults, the bacteria most commonly causing pleural empyema are $S$. aureus, S. pneumoniae, and Streptococcus pyogenes [5, 10]. Although Pneumococcal pneumonia may present with parapneumonic pleural effusions in $40 \%$ of patients, empyema occurs in only $5 \%$ of those $[5,10]$. Group A Streptococcal pneumonia occurs less frequently than Pneumococcal pneumonia but is associated with a higher frequency of pleural effusions that progress to empyema and sepsis $[10,11]$. This highlights the important role played by specific pathogens in the development and severity of empyema. Although any microorganism able to produce pneumonia could consequently progress to empyema, certain bacteria are associated with a more aggressive course. Recognizing the clinical and historic scenarios in which these microorganisms are prone to develop becomes paramount in the early detection and prompt treatment of empyema. Physicians should seek host-specific and epidemiological information that helps to identify risks of infection with specific pathogens.

A well-recognized association is that of Klebsiella pneumonia and empyema development in alcoholic patients. Their decreased host defenses impair their ability to fight the infection and usually present with advanced disease $[12,13]$. The correlation of empyema with staphylococcus genus after chest trauma or post-influenza A virus infection has been extensively described [14].

Cavitary lung disease can present as a manifestation of a wide variety of pathological processes involving the lung. Pathologically, it is described as "a gas-filled space within a zone of pulmonary consolidation or within a mass or nodule, produced by the expulsion of a necrotic part of the lesion via the bronchial tree" [15]. With infectious processes, cavitation represents the outcome of complex interactions between host and pathogen.

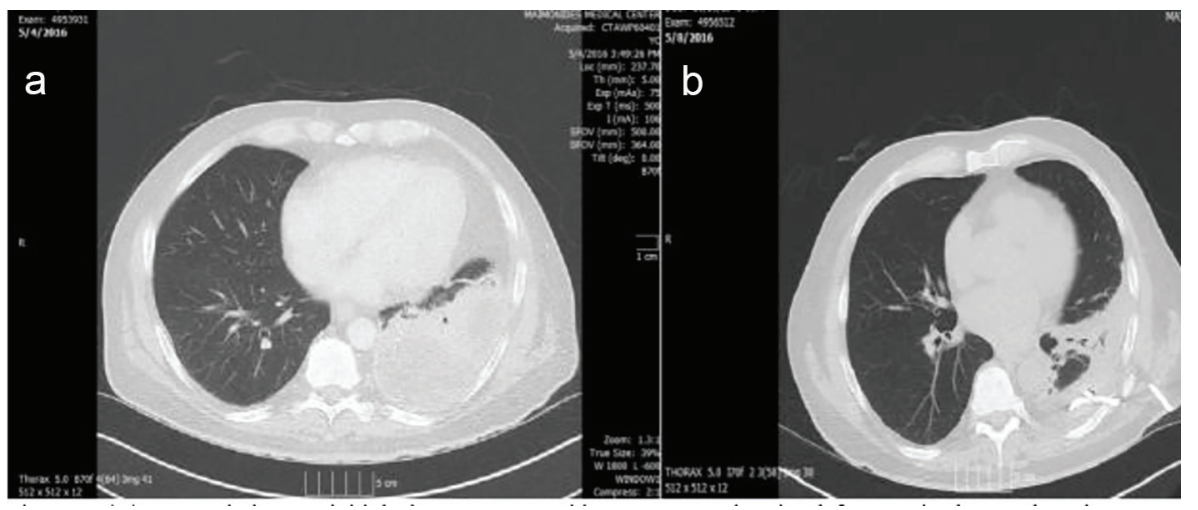

Figure 2. (A) Lung window on initial chest CT scan with IV contrast showing left posterior lower pleural space empyema and left lower lobe atelectasis. (B) Chest tube in place draining $24 \mathrm{~h}$ after admission. 


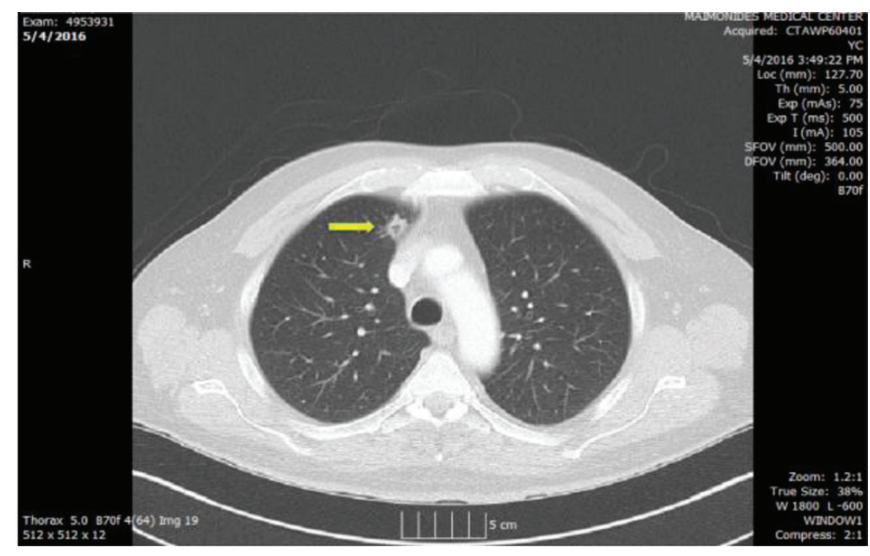

Figure 3. Chest CT showing cavitary lesion in right upper lobe (arrow) medially with irregular borders measuring $12.3 \mathrm{~mm}$.

Pulmonary actinomycosis occasionally results from the contiguous spread of infection from the neck but is usually caused by aspiration of saliva or other material containing Actinomyces [13]. This aspiration results in pneumonitis followed by local necrosis, fibrosis, and cavitation.

In this case report, we present the development of two respiratory complications including a cavitary lesion due to pulmonary actinomycosis and an empyema due to Streptococcus gordonii in an otherwise healthy middle-aged male. Microbiologic analysis by culture of both lesions demonstrated common oral flora bacteria as the pathogenic entities. This suggests the association between a recent tooth extraction and active disease process. Streptococcus gordonii is recognized as a common cause of gingivitis, tooth decay, and endocarditis but has not been previously reported as the causative organism of empyema.

We believe that this is an unusual presentation of two respiratory complications following recent dental work. We report this case with hope to remind the reader of the association between dental procedures and possible thoracic complications. Healthcare professionals should be vigilant in the prevention, early detection, and appropriate management of such conditions.

\section{Conflicts of Interest}

The authors declare that there are no conflicts of interest regarding the writing of this manuscript.

\section{References}

1. Bryant RE, Salmon CJ. Pleural empyema. Clin Infect Dis. 1996;22(5):747-762; quiz 763-744.

2. Nazemi MM, Musher DM. Empyema due to aerobic diphtheroids following dental extraction. Am Rev Respir Dis. 1973;108(5):1221-1223.

3. Jerng JS, Hsueh PR, Teng LJ, Lee LN, Yang PC, Luh KT. Empyema thoracis and lung abscess caused by viridans streptococci. Am J Respir Crit Care Med. 1997;156(5):1508-1514.

4. Ahmed RA, Marrie TJ, Huang JQ. Thoracic empyema in patients with community-acquired pneumonia. Am J Med. 2006;119(10):877-883.

5. Grijalva CG, Zhu Y, Nuorti JP, Griffin MR. Emergence of parapneumonic empyema in the USA. Thorax. 2011;66(8):663-668.

6. Heffner JE, Klein JS, Hampson C. Diagnostic utility and clinical application of imaging for pleural space infections. Chest. 2010;137(2):467-479.

7. Chung CL, Hsiao SH, Hsiao G, Sheu JR, Chen WL, Chang SC. Clinical importance of angiogenic cytokines, fibrinolytic activity and effusion size in parapneumonic effusions. PLoS One. 2013;8(1):e53169.

8. Girdhar A, Shujaat A, Bajwa A. Management of infectious processes of the pleural space: a review. Pulm Med. 2012;2012:816502.

9. Braman SS, Donat WE. Explosive pleuritis. Manifestation of group A beta-hemolytic streptococcal infection. Am J Med. 1986;81(4):723-726.

10. Lam ST, Johnson ML, Kwok RM, Bassett JT. Spontaneous bacterial empyema: not your average empyema. Am J Med. 2014;127(7):e9-e10.

11. Kaye MG, Fox MJ, Bartlett JG, Braman SS, Glassroth J. The clinical spectrum of Staphylococcus aureus pulmonary infection. Chest. 1990;97(4):788-792.

12. Tuddenham WJ. Glossary of terms for thoracic radiology: recommendations of the Nomenclature Committee of the Fleischner Society. AJR Am J Roentgenol. 1984;143(3):509-517.

13. Conant EF, Wechsler RJ. Actinomycosis and nocardiosis of the lung. J Thorac Imaging. 1992;7(4):75-84.

14. Brown JR. Human actinomycosis. A study of 181 subjects. Hum Pathol. 1973;4(3):319-330.

15. Gadkowski LB, Stout JE. Cavitary pulmonary disease. Clin Microbiol Rev. 2008;21(2):305-333, table of contents. 